\title{
Superconductivity and Mott localization
}

\author{
M. Lavagna \\ Laboratoire Louis Néel, C.N.R.S., 166 X, 38042 Grenoble-Cedex, France
}

\begin{abstract}
Résumé. - Nous développons une nouvelle approche pour traiter la localisation de Mott dans les systèmes fortement corrélés. Celle-ci est basée sur une théorie de perturbations contrainte du modèle de Hubbard, en fonction du couplage de superéchange $J$. Le terme Hartree dont nous prouvons la correspondance avec l'approximation de Gutzwiller constitue l'état fondamental naturel autour duquel fluctue le système. Nous l'interprétons en termes de résonance en complète analogie avec la résonance Abriskov-Suhl du problème Kondo. Nous montrons comment la supraconductivité est induite par les fluctuations quantiques autour de cet état résonant.
\end{abstract}

\begin{abstract}
A new approach is developed to treat the Mott localization in stronglycorrelated systems. It is based on a constrained perturbation theory of the Hubbard model as a function of the superexchange coupling $J$. The Hartree term which is proved to correspond to the Gutzwiller approximation constitutes the natural ground state around which the system fluctuates. We interpret it in terms of a resonance in complete analogy with the Abriskov-Suhl resonance of the Kondo problem. It is shown how superconductivity is induced by quantum fluctuations around this resonant state.
\end{abstract}

The recent discovery of the high superconducting temperatures in oxides has renewed the interest in the problem of magnetism and localization $[1,2]$ (in the sense of Mott) in strongly-correlated systems. $\mathrm{La}_{2} \mathrm{CuO}_{4}$ and $\mathrm{YBa}_{2} \mathrm{Cu}_{3} \mathrm{O}_{6}$ - as suggested by neutron diffraction experiments [3, 4] are antiferromagnetic insulators and superconductivity appears to be induced by doping. By the simplicity of its formulation, the Hubbard model is particularly attractive since it already contains the essential physics of the phenomenon. Before coming to the last developments, we will give a brief review of the different possible approaches :

- in the weak-coupling regime, the Coulomb interaction $U$ can be treated perturbatively leading to the Stoner mean-field theory, and the paramagnons fluctuations theory [5] which essentially corresponds to the RPA approximation. This expansion provides a correct description of magnetism and its approach, with its related excitation spectrum-magnons and paramagnons. More sophisticated theories have been developed by treating the fluctuations in a more self-consistent way [6] ;

- in the strong-coupling regime, the effect of the large Coulomb repulsion is to reduce the probability of double-occupancy and gives rise to the Mott localisation 
phenomenon: this appears at half-filling $(n=1)$ upper a critical value of $U$. Besides the spin-polaron theory [7], the more successful approach has been introduced by M. C. Gutzwiller [8, 2] and is variational. The trial wave-function $\left|\psi_{\mathrm{G}}\right\rangle$ is given by the Gutzwiller Ansatz which explicitly accounts for the reduction of probability of doubly-occupied sites :

$$
\left.\left.\left|\psi_{\mathrm{G}}\right\rangle=g^{D} \mid \text { P.S. }\right\rangle \underset{U \rightarrow \infty}{\rightarrow} P_{\mathrm{G}} \mid \text { P.S. }\right\rangle
$$

where the «parent state» P.S. is the ideal Fermi gaz;

$D$ is the number of doubly occupied sites;

$g$ is a variational parameter $(g<1)$;

and $P_{\mathrm{G}}$ is the operator which projects out the states with doubly-occupied sites.

In spite of its simplicity, the Gutzwiller Ansatz is not easy to carry on, except in some limit cases $(d=1$, small clusters...). Usually, one uses an additional approximation (also introduced by M. C. Gutzwiller) which consists to uniformly distribute the occupation probabilities on the different sites. This considerably simplifies the calculation of the ground state. Many very «physical » results are predicted by both Gutzwiller Ansatz + approximation (G.A.), notably the formation of a Mott insulator for $n=1$ and $U>U_{\mathrm{c}}$, which is found to be antiferromagnetic. Besides its successes, the Gutzwiller approach presents some weaknesses due to the non-description of the superexchange. This is to be imputed to the basically mean-field nature of the variational method, which clearly appears in the equivalent slave-boson presentation of Kotliar-Ruckenstein [9].

More recently, P. W. Anderson [10] postulated that, in some situations, the insulating ground state is no longer antiferromagnetic, but resonant among all the dimers of singlet pairs : this is the resonant valence bond (RVB) state. To formalize these ideas, he essentially proposes to modify the starting Ansatz by taking another choice of the parent state in equation (1) :

$$
\mid \text { P.S. }\rangle=\left[\sum_{\tau} a_{i}(\tau) c_{i \uparrow}^{+} c_{i+\tau \downarrow}^{+}\right]^{N / 2}|0\rangle
$$

corresponding to the formation of $N / 2$ singlet pairs distant of $\tau$ (short-range or any-range bonds). Note that a similar Ansatz has been independently proposed by Bouchaud and Lhuillier [11] in the triplet case to describe $\mathrm{He}^{3}$. This new Ansatz has the advantage of accounting for the intersite correlations which were underestimated in the Gutzwiller approach. It provides interesting results notably on the eventual superconductivity. However, the procedure remains basically variational and hard to control.

We develop here a different point of view and propose that, as the Stoner theory in the weak coupling regime, the Gutzwiller Ansatz + approximation (G.A.) is a good starting point which should be improved by adding quantum fluctuations. We present a new approach which allows to account for the fluctuations around the Gutzwiller ground state and show how this describes the intersite correlations in presence of strong on site constraints. Our main result is that this is equivalent to a constrained perturbation theory as a function of the superexchange coupling $J$, in complete analogy with the theories developed in weak coupling. 
Let us start from the formulation introduced by Kotliar-Ruckenstein [9] with the four projectors $\alpha_{i}\left(e_{i}, p_{i \sigma}\right.$ and $d_{i}$ boson operators) on the empty, singlyoccupied $\sigma$ and doubly-occupied $i$ site. The $\alpha_{i}$ fields are to be considered as markers which keep track of the site occupations. They are the ideal tools to label the averaged occupations (mean-field), and follow the dynamics of vacancies for $n \leqq 1$ (space and time-fluctuations):

$$
\begin{aligned}
H=\sum_{i j \sigma} t_{i j} z_{i \sigma}^{+} z_{j \sigma} c_{i \sigma}^{+} c_{j \sigma}+U \sum_{i} d_{i}^{+} d_{i} & \\
& +\sum_{i} \lambda_{i}^{(1)}\left(e_{i}^{+} e_{i}+\sum_{\sigma} p_{i \sigma}^{+} p_{i \sigma}+d_{i}^{+} d_{i}^{-1}\right) \\
& +\sum_{i \sigma} \lambda_{i \sigma}^{(2)}\left(c_{i \sigma}^{+} c_{i \sigma}-p_{i \sigma}^{+} p_{i \sigma}-d_{i}^{+} d_{i}\right)
\end{aligned}
$$

with

$$
z_{i \sigma}=e_{i}^{+} p_{i \sigma}+p_{i-\sigma}^{+} d_{i}
$$

$\lambda_{i}^{(1)}$ and $\lambda_{i \sigma}^{(2)}$ are Lagrange multipliers to enforce the constraints :

$$
e_{i}^{2}+\sum_{\sigma} p_{i \sigma}^{2}+d_{i}^{2}=1 \quad \text { and } \quad n_{i \sigma}=p_{i \sigma}^{2}+d_{i}^{2}
$$

Expressions (3) and (4) reflects the three classes of hopping:

- hopping between singly occupied and empty sites represented by the $e^{2} p^{2}$ term (electrons motion);

- hopping between singly and doubly occupied sites represented by the $p^{2} d^{2}$ term (holes motion);

- hopping between singly occupied $\uparrow$ and $\downarrow$ sites $\left(e p^{2} d\right.$ term) responsible for the exchange when virtually considered through the doubly occupied sites.

(3) is strictly equivalent to the Hubbard model with $z_{i \sigma}$ specified by (4). However, the choice of $z_{i \sigma}$ is not unique, and one can replace $z_{i \sigma}$ by any combinations $U_{i} z_{i \sigma} V_{i}$ where $U_{i}$ and $V_{i}$ are diagonal operators such as $U_{i}=1$ in the $(0)$ and $(-\sigma)$ configurations and $V_{i}=1$ in $(+\sigma)$ and $(\uparrow \downarrow)[1]$. One can in particular choose [9] :

$$
\bar{z}_{i \sigma}=\left(1-d_{i}^{+} d_{i}-p_{i \sigma}^{+} p_{i \sigma}\right)^{-1 / 2} z_{i \sigma}\left(1-e_{i}^{+} e_{i}-p_{i-\sigma}^{+} p_{i-\sigma}\right)^{-1 / 2} .
$$

All the choices are formally equivalent as far as the constraints are exactly satisfied at each site at any time. Unfortunately, this is no longer true as soon as one makes approximations. Typically, the mean-field (MF) approximation makes the constraint satisfied on the average, and leads to different results depending on the special choice of $z_{i \sigma}$. These discrepancies are supposed to disappear if one succeeds in going beyond MF as proposed in this paper. When stopping at MF, the choice (5) is more judicious since it renormalizes $z_{i \sigma}$ by its value in the uncorrelated case, and so gives back the free electron gaz for $U=0$. For this choice, it has been shown [9] that the saddle-point approximation on the boson fields is equivalent to the Gutzwiller approximation. The merit of the slave-boson formulation is to transform the variational procedure into a standard field theory problem and so to open the way to further developments. 
By minimizing the mean-field free energy with respect to the 4 slave-bosons, and 3 Lagrange multipliers, one gets 7 saddle-point equations (including the 3 constraints) which permit to determinate the number of doubly-occupied sites $d_{0}^{2}$ (to which $e_{0}^{2}$ and $p_{0 \sigma}^{2}$ are related through the constraints) and so the factor of band renormalization $q_{0}=z_{0}^{2}$. We have checked that this gives back the Gutzwiller relations [8, 12] that we recall below. Moreover, we emphasize here that the content of the saddle-point equations is even richer, and can also lead to the determination of the Lagrange multipliers. In principle, the resolution can be done at any point $(U, n)$. However, we give here the results only for the two interesting regimes I and II. Noting $\left\langle\sum_{i j} t_{i j} c_{i \sigma}^{+} c_{j \sigma}\right\rangle=\varepsilon_{\sigma}=\bar{\varepsilon}_{0} / 2=-U_{\mathrm{c}} / 16$ in the paramagnetic half-filled band case :

regime I : $\left(u=U / U_{\mathrm{c}}<1\right.$ and $\left.n=1\right)$ :

$$
\begin{aligned}
& d_{0}^{2}=(1-u) / 4 \text { and } q_{0}=z_{0}^{2}=1-u^{2} \\
& \lambda_{0}^{(1)}=U_{\mathrm{c}}(1+u)(2-u) / 4 \text { and } \lambda_{0}^{(2)}=U / 2 .
\end{aligned}
$$

The Mott transition $\left(q_{0} \rightarrow 0\right)$ naturally arises for $U>U_{\mathrm{c}}$,

regime II : $\left(u=U / U_{\mathrm{c}}>1\right.$ and $\left.|1-n|=\delta \ll 1\right)$ :

$$
d_{0}^{2}=\delta(1-\zeta)^{2} / 4 \zeta \text { and } q_{0}=2 \delta / \zeta
$$

with the notation $\zeta=\sqrt{1-1 / u}$

$$
\lambda_{0}^{(1)}=\lambda_{0}^{(2)}=U(1 \pm \sqrt{1-1 / u}) / 2
$$

(+ and - for $n>1$ and $n<1$ respectively).

These results take all their physical meaning if realize that $\lambda_{0}^{(2)}$ is nothing else as a chemical potential. It fixes the position of the narrow band and gives rise to a gap (Mott gap) for $U>U_{\mathrm{c}}$ of width $U \sqrt{1-U_{\mathrm{c}} / U}$. By analogy with the Abrikosov-Suhl resonance of the Kondo problem, we propose to interpret the renormalization of the band as a resonance pinned at the Fermi level, and cut by a gap for $U>U_{\mathrm{c}}$ as for the Kondo lattice. This resonance comes from the virtual excitations of electrons between different sites in the strong coupling regime of the Hubbard model, in the same way as the Abrikosov-Suhl resonance comes from the virtual excitations between extended $\mathrm{c}$ and localized $\mathrm{f}$ electrons in the strong coupling limit of the Anderson model $\left(n_{\mathrm{f}}=1\right)$. Both resonances are typical of almost-localized systems. It is probable that they play an essential role to renormalize (Anderson-Morel scaling [13]) the eventual superconducting paring arising from quantum fluctuations, in order to gain the direct intersite Coulomb repulsion. As for the Kondo problem, the « real » electron band would present additional queue of unrenormalized density on each site of the resonance.

Our purpose now is to go beyond this saddle-point approximation and include quantum fluctuations. We postulate that, along the Mott transition line $(n=1)$ and for strong-coupling $\left(U \gg U_{c}\right)$, the slave-boson-Gutzwiller procedure is equivalent to a constrained perturbation theory in $J$. We do not want to give here the details of the demonstration which can be found elsewhere [14]. Let us 
just say that, in the considered regime, the intersite correlations are dominated by the $d$ - $d$ slave-boson exchange which is shown to coincide to the usual superexchange coupling $J=2 t^{2} / U$. Due to a gauge-invariance property of the Hamiltonian, one can incorporate the phase-part of the $d$ - $d$ boson propagator into the Lagrange multiplier $\lambda^{(2)}$. In this radial gauge, the propagator takes the following matricial $d x \lambda$ representation:

$$
D_{0}^{-1}=\left(\begin{array}{cc}
1 / J & d_{0} \\
d_{0} & 0
\end{array}\right)
$$

This corresponds to the intersite exchange coupling added to the large on-site constraints. We emphasize the essential role played by the $\lambda^{(2)}$ intèraction. While it is responsible for the mean-field symmetry breaking (see above the formation of the Mott gap), its fluctuations are essential in order to restore the brokensymmetry. The fluctuations associated to the coupled radial-phase $J-\lambda^{(2)}$ interaction can then be treated within the usual many body methods. We propose the RPA approximation (ring + ladder diagrams) which is an extension of the $1 / N$ expansion. This leads to a $d$-wave superconductivity instability close to the Mott transition. The physical origin of this superconductivity is due to intersite interactions combined with large on-site constraints. It is close to the situation found in the $1 / N$ expansion of the Anderson model [15]. We stress that the difference in the cut-off energy scales ( $q W$ instead of the Kondo temperature $T_{\mathrm{K}}$ ) is a factor which can explain the high temperature $T_{\mathrm{c}}$ observed in the new oxides.

In conclusion, we have sketched here a new kind of study of the Mott transition in the strong-coupling limit of the Hubbard model. The development that we proposed gives an insight into the deep similitude between these systems and Heavy Fermions. The approach to the localization which is inherent to both situations is described in a very parallel way :

- mean-field broken-symmetry associated to a resonant state ;

- symmetry restored by quantum fluctuations, representing intersite correlations combined with large on-site constraints. This cleared up the onset of superconductivity close to the localization transition. It is probable that the quantum fluctuations may have many other important consequences [14] which may be related to the presence of this mysterious RVB state.

\section{References}

[1] For a very complete review, see the series of lectures given by P. NoziÈres : Magnetism and localization in Fermi liquids (Collège de France) 1986 (unpublished).

[2] Vollhardt, D., Rev. Mod. Phys. 56 (1984) 99 (and references within).

[3] Shirane, G., Endoh, Y., Birgeneau, R. J., Kastner, M. A., Hidaka, Y., Oda, M., Suzuki, M., Marakami, T., Phys. Rev. Lett. 59 (1987) 1643.

[4] Tranouada, J. M., Cox, D. E., Kunnmann, W., Moudden, H., Shirane, G., Suenaga, M., Zollinker, P., WaKmin, D., Sinha, S. K., Alvarez, M. S., Jacobson, A. K., Johnston, D. C., Phys. Rev. Lett. 60 (1988) 156 ; 
Rossat-Mignot, J., Burlet, P., Jurgens, M. J. G. M., Henry, J. Y. and VetTier, C., Physica C 152 (1988) 19.

[5] Berk, N. F., Schrieffer, J. R., Phys. Rev. Lett. 17 (1966) 433 ;

Doniach, S., Engelsberg, S., Phys. Rev. Lett. 17 (1966) 750.

[6] MoriYA, T., Electron correlation and magnetism in narrow band systems (Berlin Springer) 1980 (and references within).

[7] Montambaux, G., Héritier, M., Lederer, P., J. Low Tem. Phys. 47 (1982) 39 and references within.

[8] Gutzwiller, M. C., Phys. Rev. Lett. 10 (1963) 159 and Phys. Rev. A 134 (1964) 923 and 137 (1965) 1726 ;

Brinkman, W. F. and Rice, T. M., Phys. Rev. B 2 (1970) 4302 ;

Rice, T. M. and Brinkman, W. F., Phys. Rev. B 5 (1972) 4350.

[9] Kotliar, G. and Ruckenstein, A. E., Phys. Rev. Lett. 57 (1986) 1362.

[10] Anderson, P. W., Science 235 (1987) 1196 ;

Baskaran, G., Zou, Z., Anderson, P. W., Solid State Commun. 63 (1987) 973.

[11] Bouchaud, J. P. and Lhuillier, C., Europhys. Lett. 3 (1987) 1273.

[12] Vollhardt, D., Wolfe, P. and Anderson, P. W., Phys. Rev. B 35 (1987) 6703.

[13] Morel, P. and Anderson, P. W., Phys. Rev. 125 (1962) 1263.

[14] Lavagna, M. (to be published).

[15] Lavagna, M., Millis, A. J. and Lee, P. A., Phys. Rev. Lett. 58 (1987) 266. 